\title{
Continuous fat-free mass decline in COPD: fact or fiction?
}

\section{To the Editor:}

Patients with chronic obstructive pulmonary disease (COPD) generally have lower fat-free mass (a surrogate marker of skeletal muscle mass) compared with their healthy peers $[1,2]$, and this is associated with decreased functional capacity [3], the presence of other comorbidities (e.g. osteoporosis and renal impairment) [4] and increased mortality risk [5]. To date, longitudinal observational studies have shown no difference in the mean decline in fat-free mass over time between COPD patients and non-COPD control subjects $[6,7]$. However, it remains unknown whether and to what extent a subgroup of COPD patients suffer continuous loss of fat-free mass. We hypothesised that COPD patients have 1) a higher prevalence of continuous fat-free mass decline, and 2) that this subgroup has a steeper decline of fat-free mass compared with a non-COPD control group with a continuous fat-free mass decline. We tested this hypothesis in the ECLIPSE (Evaluation of COPD Longitudinally to Identify Predictive Surrogate Endpoints) study, which had a 3 year follow-up period.

The design, aims and methodology of ECLIPSE have been described elsewhere [8]. Fat-free mass was measured by a trained research nurse using bio-electrical impedance (Bodystat 1500; Bodystat Ltd, Douglas, UK) (each centre had the same device) after at least $4 \mathrm{~h}$ fasting and calculated with a COPD specific formula [9]. Only subjects with complete fat-free mass data throughout the study were included in the present analysis. Low attenuation was determined by a chest computed tomography scan. Decline was defined as any fat-free mass measurement during follow-up that was lower than the fat-free mass of the previous year, and the number of years with a fat-free mass decline was summed. The prevalence of 3-year fat-free mass decline was calculated. Severe (hospitalisations) and moderate (use of corticosteroids/ antibiotics) exacerbations during the 3 year follow-up were recorded. Frequent exacerbators were defined as those patients with at least two exacerbations per year in the 3 year follow-up. The Chi-squared test was used to test the prevalence of fat-free mass decline between the COPD patients and the non-COPD control group. The unpaired t-test was used to compare the characteristics and level of decline in the patient group with the control group, and the characteristics in the group with a continuous decline compared with the remaining group. A p-value $<0.05$ was considered statistically significant.

In total, 1494 COPD and 497 control subjects (both smoking and non-smoking) were included in this analysis. The control group was younger (mean \pm SD $55 \pm 9$ versus $63 \pm 7$ years; $\mathrm{p}<0.05$ ), included more women (51\% versus $45 \%$; $\mathrm{p}<0.05$ ), had higher lung function (mean \pm SD forced expiratory volume in $1 \mathrm{~s}: 111.3 \pm 13.3$ versus $49.9 \pm 15.4 \%$ predicted; $\mathrm{p}<0.01$ ), and had a comparable fat-free mass index (mean \pm SD $18.2 \pm 2.3$ versus $\left.18.1 \pm 2.5 \mathrm{~kg} \cdot \mathrm{m}^{-2} ; \mathrm{p}>0.05\right)$ when compared with the patients. More patients had a decline in fat-free mass from baseline to year one compared with controls ( $50 \%$ versus $39 \%$, respectively; $\mathrm{p}<0.01$ ), but this effect was not present when comparing the data of year 1 with year 2 (52\% versus $49 \%$, respectively; $\mathrm{p}=0.30)$ and of year 2 with year 3 ( $51 \%$ versus $54 \%$, respectively; $\mathrm{p}=0.25)$. The percentage of subjects with three consecutive years of fat-free mass decline was low, but significantly higher in the patients than in controls ( $7 \%$ versus $5 \%$; $\mathrm{p}=0.04)$. The level of fat-free mass decline tended to differ between patients and controls who had continuous fat-free mass decline (median (interquartile range) $-3.29(-5.03--2.14)$ versus $-2.61(-3.65-$ $-1.85) \mathrm{kg}$, respectively; $\mathrm{p}=0.05$ ). Gender, age, lung function, cumulative smoking exposure (pack-years) and body mass index (BMI) in patients with continuous fat-free mass decline was similar to the remaining group of patients, but they tended to have a higher fat-free mass index (table 1). In addition, besides comparable baseline low attenuation area, change in low attenuation area over the 3 years was higher in the patients with continuous fat-free mass decline compared with the remaining patients. Also, the total number of exacerbations during follow-up was higher in the patients with continuous fat-free mass decline compared with the remaining patients (median (interquartile range) $3(1-5)$ versus $2(1-5) ; p=0.04)$. However, the prevalence of frequent exacerbators was comparable between the groups ( $10 \%$ versus $9.7 \%$; $\mathrm{p}=0.93$ ).

The main results show that the prevalence of continuous fat-free mass decline was small, but higher in COPD patients compared with a non-COPD control group. Nevertheless, the level of fat-free mass decline in the subjects with a 3-year continuous fat-free mass decline was not different between the groups. The patients with a continuous fat-free mass decline had more exacerbations.

In the Health $\mathrm{ABC}$ (Health, Aging and Body Composition) study, the 7 year decline in lean mass was comparable between patients with obstructive lung disease and control subjects [6]. These observations 
TABLE 1 Characteristics of the patients with or without continuous fat-free mass decline

Continuous fat-free mass decline

Remaining patients

Males \%
Age years
FEV $1 \%$ predicted
FEV $1 / F V C \%$ predicted
Smoking history pack-years
BMI $\mathrm{kg} \cdot \mathrm{m}^{-2}$
FFMI $\mathrm{kg} \cdot \mathrm{m}^{-2}$
Low attenuation area HU
Change in low attenuation area $\Delta \mathrm{HU}$

66

$63.4 \pm 6.2$

$48.8 \pm 15.0$

$57.9 \pm 16.0$

$45.0(28.8-56.3)$

$26.7 \pm 5.3$

$18.5 \pm 2.4$

$11.4(3.9-22.0)$

$1.6(-0.3-5.9)$
65

$63.0 \pm 7.1$

$49.9 \pm 15.4$

$59.7 \pm 14.6$

$43.0(30.0-57.0)$

$26.6 \pm 5.6$

$18.0 \pm 2.5^{\#}$

$16.5(4.9-24.1)$

$1.0(-0.8-3.8)^{*}$

Data are presented as $\%$, mean \pm SD or median (interquartile range). $F E V_{1}$ : post-bronchodilator forced expiratory volume in $1 \mathrm{~s}$; FVC: forced vital capacity; FEV1/FVC: tiffeneau index; BMI: body mass index; FFMI: fat-free mass index. ${ }^{\#}: p=0.05 ;{ }^{*}: p<0.05$.

were reproduced in the ECLIPSE study [7]. Our study extends these previous observations by investigating the prevalence and correlates of patients with continuous fat-free mass decline, often termed muscle wasting [10]. Since the prevalence of abnormal low muscle mass is highly prevalent in cross-sectional COPD studies, it is often assumed that this is the consequence of muscle wasting. The current results show that the prevalence of continuous fat-free mass decline is infrequent in COPD patients, but it is commoner among our COPD population than in the controls ( $7 \%$ versus $5 \%$, respectively). These differences are too small to affect the overall rate of fat-free mass decline. Nevertheless, our data indicate that there is a small subgroup of patients with disease-specific muscle wasting defined by continuous fat-free mass decline, which might be partly explained by excess exacerbation count, an observation in line with previous reports [11]. Alternatively, our findings are also in line with a genetic predisposition for the development of a thin body habitus in a subgroup of COPD patients [12], but this does not fully explain the high prevalence of low muscle mass in COPD patients. Additional causes seem responsible for this phenotype. For instance, as recently shown in the Baltimore Longitudinal Study of Aging [13], low body weight can be a risk factor for developing COPD. Further, an association between low BMI and increased risk for COPD has been confirmed in never-smokers in the BOLD (Burden of Obstructive Lung Disease) study [14]. An alternative hypothesis might be the weight lowering effect of tobacco smoke, which is concurring with the development of COPD [15]. Future research should discriminate between COPD patients with low muscle mass due to a constitutionally low fat-free mass versus those suffering from true muscle wasting. As a limitation, we acknowledge that the bio-electrical impedance analysis is not the gold standard for longitudinal changes of body composition although its validity in subjects with a normal hydration state is confirmed [16].

In summary, our results show that only a small proportion of COPD patients suffer continuous decline of fat-free mass. Additional causes are probably responsible for the high prevalence of low muscle mass in COPD.

$\boldsymbol{0}$

@ERSpublications

Only a small proportion of COPD patients suffer continuous decline of fat-free mass http://ow.ly/R6ioA

Erica P.A. Rutten ${ }^{1,2}$, Martijn A. Spruit ${ }^{1,3}$, Merry-Lynn N. McDonald ${ }^{4}$, Stephen Rennard ${ }^{5}$, Alvar Agusti, ${ }^{6,7}$, Bartolome Celli ${ }^{8}$, Bruce E. Miller ${ }^{9}$, Courtney Crim ${ }^{10}$, Peter M.A. Calverley ${ }^{11}$, Corrine Hanson ${ }^{12}$, William MacNee ${ }^{13}$, Frits M.E. Franssen ${ }^{1}$, Lowie Vanfleteren ${ }^{1}$ and Emiel F.M. Wouters ${ }^{1,2}$ on behalf of the ECLIPSE (Evaluation of COPD Longitudinally to Identify Predictive Surrogate End-points) Investigators

${ }^{1}$ Dept of Research and Education, CIRO, Horn, The Netherlands. ${ }^{2}$ Maastricht University Medical Center, Maastricht, The Netherlands. ${ }^{3}$ REVAL - Rehabilitation Research Center, BIOMED - Biomedical Research Institute, Faculty of Medicine and Life Sciences, Hasselt University, Diepenbeek, Belgium. ${ }^{4}$ Channing Division of Network Medicine, Harvard Medical School, Brigham and Women's Hospital, Boston, MA, USA. ${ }^{5}$ Division of Internal Medicine, University of Nebraska, Nebraska Medical Center, Omaha, NE, USA. ${ }^{6}$ Thorax Institute, Hospital Clinic, IDIBAPS, University of Barcelona, Barcelona, Spain. ${ }^{7}$ CIBERES, Madrid, Spain. ${ }^{8}$ Pulmonary and Critical Care Medicine, Brigham and Women's Hospital, Harvard Medical School, Boston, MA, USA. ${ }^{9}$ GlaxoSmithKline, King of Prussia, PA, USA. ${ }^{10}$ GlaxoSmithKline, Research Triangle Park, NC, USA. ${ }^{11}$ The University of Liverpool, Liverpool, UK. ${ }^{12}$ Medical Nutrition Education, University of Nebraska, Nebraska Medical Center, Omaha, NE, USA. ${ }^{13}$ The University of Edinburgh, Edinburgh, UK.

Correspondence: Erica P.A. Rutten, Dept of Research and Education, Centre of expertise for chronic organ failure (CIRO+), Postbus 4009, 6080 AA Haelen, The Netherlands. E-mail: ericarutten@ciro-horn.nl

Received: May 042015 | Accepted after revision: July 092015 | First published online: Sept 172015 
Support statement: the ECLIPSE study was sponsored by GlaxoSmithKline. The study sponsor did not place any restrictions on statements made in the final paper. Funding information for this article has been deposited with FundRef.

Conflict of interest: Disclosures can be found alongside the online version of this article at erj.ersjournals.com

This article has been revised according to the correction published in the November 2016 issue of the European Respiratory Journal.

\section{References}

1 Rutten EP, Bakke P, Pillai S, et al. The association between body composition and self-reported co-morbidity in subjects with chronic obstructive pulmonary disease. Open J Intern Med 2012; 2: 100-106.

2 Vestbo J. Clinical assessment, staging, and epidemiology of chronic obstructive pulmonary disease exacerbations. Proc Am Thorac Soc 2006; 3: 252-256.

3 Seymour JM, Spruit MA, Hopkinson NS, et al. The prevalence of quadriceps weakness in COPD and the relationship with disease severity. Eur Respir J 2010; 36: 81-88.

4 Vanfleteren LE, Spruit MA, Groenen M, et al. Clusters of comorbidities based on validated objective measurements and systemic inflammation in patients with chronic obstructive pulmonary disease. Am J Respir Crit Care Med 2013; 187: 728-735.

5 Vestbo J, Prescott E, Almdal T, et al. Body mass, fat-free body mass, and prognosis in patients with chronic obstructive pulmonary disease from a random population sample: findings from the Copenhagen City Heart Study. Am J Respir Crit Care Med 2006; 173: 79-83.

6 van den Borst B, Koster A, Yu B, et al. Is age-related decline in lean mass and physical function accelerated by obstructive lung disease or smoking? Thorax 2011; 66: 961-969.

7 Rutten EPA, Calverley PM, Casaburi R, et al. Changes in body composition in patients with chronic obstructive pulmonary disease: do they influence patient related outcomes? Ann Nutr Metab 2013; 63: 239-247.

8 Vestbo J, Anderson W, Coxson HO, et al. Evaluation of COPD Longitudinally to Identify Predictive Surrogate End-points (ECLIPSE). Eur Respir J 2008; 31: 869-873.

9 Steiner MC, Barton RL, Singh SJ, et al. Bedside methods versus dual energy X-ray absorptiometry for body composition measurement in COPD. Eur Respir J 2002; 19: 626-631.

10 Anker SD, Coats AJ, Morley JE, et al. Muscle wasting disease: a proposal for a new disease classification. J Cachexia Sarcopenia Muscle 2014; 5: 1-3.

11 Hopkinson NS, Tennant RC, Dayer MJ, et al. A prospective study of decline in fat free mass and skeletal muscle strength in chronic obstructive pulmonary disease. Respir Res 2007; 8: 25.

12 Wan ES, Cho MH, Boutaoui N, et al. Genome-wide association analysis of body mass in chronic obstructive pulmonary disease. Am J Respir Cell Mol Biol 2011; 45: 304-310.

13 Harik-Khan RI, Fleg JL, Wise RA. Body mass index and the risk of COPD. Chest 2002; 121: 370-376.

14 Lamprecht B, McBurnie MA, Vollmer WM, et al. COPD in never smokers: results from the population-based burden of obstructive lung disease study. Chest 2011; 139: 752-763.

15 Seeley RJ, Sandoval DA. Neuroscience: weight loss through smoking. Nature 2011; 475: 176-177.

16 Kyle UG, Bosaeus I, De Lorenzo AD, et al. Bioelectrical impedance analysis - part I: review of principles and methods. Clin Nutr 2004; 23: 1226-1243.

\section{Diagnosis of COPD before cardiac surgery}

To the Editor:

We recently performed a retrospective study of the utility of respiratory function tests prior to cardiac surgery at our hospital. The study aimed to identify whether an association exists between pre-operative respiratory function tests and post-operative outcomes. The study also aimed to determine how the definition of chronic obstructive pulmonary disease (COPD) by Global Initiative in Chronic Obstructive Lung Disease (GOLD) [1] or by lower limit of normal (LLN) [2] may alter peri-operative management.

Between January 2011 and January 2014, 1330 patients underwent cardiac surgery at Holy Spirit Northside Private Hospital (HSNPH), Brisbane, Australia, a private hospital facility operating as part of the St Vincent's Health Australia group. Of this number, 289 mainly symptomatic patients were referred for respiratory function tests which were performed at HSNPH within 3 days of admission. The patients then proceeded with the intended cardiac surgery.

A patient was classified as COPD (GOLD) if the ratio of forced expiratory volume (FEV1) to forced vital capacity (FVC) was $<0.70$ [1]. A patient was classified as COPD (LLN) if the FEV1/FVC ratio was <LLN. The LLN criteria identify the fifth percentile of the study population, defined as 1.645 relative standard deviations below the mean predicted value [2]. The Global Lung Function Initiative 2012 equations were used to calculate predicted values and z-scores for spirometry [3]. 